OPEN ACCESS

Edited by:

Gustavo Henrique Goldman,

University of São Paulo, Brazil

Reviewed by:

Chengshu Wang,

Shanghai Institutes for Biological Sciences (CAS), China

Drauzio Eduardo Naretto Rangel, Universidade Brasil, Brazil

*Correspondence:

Weiguo Fang

wfang1@zju.edu.cn

Specialty section:

This article was submitted to Fungi and Their Interactions,

a section of the journal

Frontiers in Microbiology

Received: 10 December 2018 Accepted: 28 February 2019

Published: 20 March 2019

Citation:

Wu C, Zhang X and Fang W (2019) Increasing Pyruvate Concentration Enhances Conidial Thermotolerance in the Entomopathogenic Fungus Metarhizium robertsii.

Front. Microbiol. 10:519. doi: 10.3389/fmicb.2019.00519

\section{Increasing Pyruvate Concentration Enhances Conidial Thermotolerance in the Entomopathogenic Fungus Metarhizium robertsii}

\author{
Congcong Wu, Xing Zhang and Weiguo Fang* \\ MOE Key Laboratory of Biosystems Homeostasis and Protection, Institute of Microbiology, Zhejiang University, \\ Hangzhou, China
}

The fungal entomopathogens Metarhizium spp. have been developed as environmentally friendly mycoinsecticides. However, heat stress severely reduces the viability of Metarhizium conidia in the field, which is an important obstacle to the successful use of these mycoinsecticides. Heat treatment induces rapid accumulation of pyruvate, which timely scavenges heat-induced ROS (reactive oxygen species) in hyphal cells of $M$. robertsii. However, in heat-treated conidia, pyruvate accumulation occurs later than the rapid production of ROSs, which could harm the conidial cells. In the present study, a transgenic $M$. robertsii strain was constructed with the pyruvate kinases gene overexpressed during conidiation. Two independent transformants of the transgenic strain produced conidia under optimal conditions with elevated pyruvate concentration. This inhibits the rapid heat-induced ROS production and prevents the collapse of mitochondrial membrane potential, thereby increasing conidial tolerance to heat stress. In conclusion, the tolerance of $M$. robertsii conidia to heat stress was improved by increasing the conidial pyruvate concentration, which could be translated into a more effective pest control.

Keywords: Metarhizium, heat stress, pyruvate accumulation, biological control, entomopathogenic fungi

\section{INTRODUCTION}

The entomopathogenic fungi Metarhizium spp. are being developed as environmentally friendly mycoinsecticides ( $\mathrm{Li}$ et al., 2010). The conidium is the propagule that initiates pathogenesis, and it is thus the acting component in the mycoinsecticides. In spite of Metarhizium being adaptable to many environments, abiotic stresses, such as heat and UV radiation, severely reduce the viability of conidia in the field, and these stressors are important obstacles to the successful use of Metarhizium spp. (Fang et al., 2012; Liao et al., 2014; Zhao et al., 2016). Conidial tolerance to abiotic stresses can be improved by selecting the optimal growth substrate and conditions for conidial production (Rangel et al., 2015). Based on the molecular mechanisms of abiotic stress tolerance, conidial tolerance to abiotic stresses can also be achieved by genetic engineering. Reactive oxygen species (ROSs) are upregulated by multiple stresses, and ROS scavengers reduce the level of stress-induced ROSs and are thus involved in stress tolerance. Overexpressing genes that encode a SOD (superoxide dismutase) and a bacterial thioredoxin increases heat tolerance in the entomopathogenic fungus Beauveria bassiana (Xie et al., 2010; Ying and Feng, 2011). The expression of the small heat shock protein HSP25 is upregulated when $M$. robertsii is grown at extreme temperatures or in the presence of oxidative or osmotic agents. Overexpressing HSP25 increases the growth of M. robertsii under heat 
stress and enhances the tolerance of heat shock-treated conidia to osmotic stress (Liao et al., 2014). It is likely that the discovery of other mechanisms of heat stress tolerance would provide more approaches for the further improvement of mycoinsecticides.

Recently, it was found that pyruvate is a fungal ROS scavenger. In heat treated hyphae, pyruvate accumulation is coincident with ROS production, and pyruvate timely scavenges heatinduced ROSs, thereby reducing the damage caused by ROSs to cellular proteins and the mitochondrial membrane potential. Heat treatment also upregulates the production of ROSs and pyruvate in the conidia, but pyruvate accumulation occurs later than ROS burst, and the ROS level thus remains high until pyruvate accumulation (Zhang et al., 2017). As pyruvate accumulation functions as the first line of defense against heatinduced ROSs in conidia (Zhang et al., 2017), it is likely that the ROSs cause damages to the conidia before pyruvate accumulation. In present study, we increased the pyruvate concentration in unstressed conidia by overexpressing a pyruvate kinase gene. This reduced the level of heat-induced ROSs and consequently improved conidial tolerance to heat stress.

\section{RESULTS}

\section{Increasing Pyruvate Concentration in the Conidia by Overexpressing a Pyruvate Kinase Gene}

In our previous study, we found that heat treatment induced pyruvate accumulation in $M$. robertsii by upregulating the pyruvate kinase gene (MAA_06851) for pyruvate production and by downregulating many genes involved in pyruvate consumption, including MAA_02871 and MAA_08787 (Zhang et al., 2017). The pyruvate kinase (MAA_06851) catalyzes the transfer of a phosphate group from phosphoenolpyruvate to adenosine diphosphate, yielding pyruvate and ATP. MAA_02871 encodes a pyruvate transporter that moves pyruvate from the cytoplasm into mitochondria for the TCA cycle, while MAA_08787 encodes the $\beta$-subunit of the pyruvate dehydrogenase E1 component. To increase the pyruvate concentration in the conidia that were grown under the optimal conditions [on potato dextrose agar (PDA) at $26^{\circ} \mathrm{C}$ ], we overexpressed the pyruvate kinase gene (MAA_06851) or knocked down using the antisense RNA method the two genes (MAA_02871 and MAA_08787) that are involved in pyruvate consumption. Pyruvate is located at a key intersection in the network of metabolic pathways, so constitutive modification of pyruvate metabolic pathway could harm some fungal development stages. Meanwhile, as described above, conidium is the acting component in mycoinsecticides, and so we intended to increase pyruvate concentration only in conidia to overcome the time difference between the heat-induced ROS production and pyruvate accumulation and enhance conidial heat tolerance. To do this, the three genes described above were controlled by the conidiation specific promoter of the polyketide synthase gene $P k s 1$, which is only highly expressed during conidiation and involved in synthesis of conidial pigments (Zeng et al., 2017, 2018).

For the two genes involved in pyruvate consumption, no significant differences in the expression levels during conidiation were observed between the wild-type (WT) strain and the five randomly selected transformants $(p>0.05$, Tukey's test in one-way ANOVA), indicating failure of gene expression suppression using the antisense RNA method. However, we obtained the transgenic strain T-MAA_06851 ${ }^{O E}$ with the pyruvate kinase gene (MAA_06851) overexpressed in the conidiating mycelium that was grown PDA for 5 days at optimal temperature $\left(26^{\circ} \mathrm{C}\right)$ (Figure 1A). Two independent transformants (T-MAA_06851 ${ }^{O E}-1$ and T-MAA_06851 $\left.{ }^{O E}-2\right)$ were subjected to the pyruvate assay. Grown under the optimal conditions (at $26^{\circ} \mathrm{C}$ on PDA for 14 days), the pyruvate concentrations in the conidia of the two transformants were significantly higher than the WT strain $(p<0.05$, Tukey's test in the one-way ANOVA). No significant difference was found between these two transformants $(p>0.05$, one-way ANOVA) (Figure 1B). The pyruvate concentrations in the conidia of the transformants grown under the optimal conditions were comparable to the WT conidia that had been heat treated ( $p>0.05$, Tukey's test in the one-way ANOVA). However, heat treatment did not further increase the pyruvate concentration in the conidia of the transformants $(p>0.05$, Tukey's test in the one-way ANOVA) (Figure 1B).

\section{Increasing the Pyruvate Concentration Reduces the Level of Total Ross and Prevents the Heat-Induced Collapse of Mitochondrial Membrane Potential in Conidia}

We then investigated whether elevating pyruvate concentration could reduce the ROS level in heat-treated conidia. At the optimal temperature $\left(26^{\circ} \mathrm{C}\right)$, no significant difference in the ROS level was found between WT and the two transformants $\left(T-M A A \_06851^{O E}-1\right.$ and T-MAA_06851 $\left.{ }^{O E}-2\right)(p>0.05$, Tukey's test in one-way ANOVA). In previous studies (e.g., Fang et al., 2010; Rangel et al., 2015), $42^{\circ} \mathrm{C}$ was often used to assay heat tolerance of Metarhizium conidia in context of development of mycoinsecticides. In this study, we thus used $42^{\circ} \mathrm{C}$ to assay the heat tolerance of the transformants. The heat treatment $\left(42^{\circ} \mathrm{C}\right.$ for $10 \mathrm{~min}$ ) significantly increased the ROS level in WT conidia $(p<0.05$, Tukey's test in one-way ANOVA), but this did not affect the level of ROSs in the two transformants $(p>0.05$, Tukey's test in one-way ANOVA) (Figure 2A). The ROS level in the heat-treated WT conidia was thereby significantly higher than the two transformants, which were not significantly different from each other. The elongated heat treatment $\left(42^{\circ} \mathrm{C}\right.$ for $\left.30 \mathrm{~min}\right)$ also significantly increased the ROS level in the two transformants, which were still significantly lower than the WT strain $(p<0.05$, Tukey's test in one-way ANOVA) (Figure 2A).

ROSs destabilizes the mitochondrial membrane potential (Wang et al., 2007). At the optimal temperature $\left(26^{\circ} \mathrm{C}\right)$, the ratio of green/red fluorescence intensity in the WT conidia was not significantly different from the two transformants $\left(T-M A A \_06851^{O E}-1\right.$ and T-MAA_06851 ${ }^{O E}-2$; Figures 2B,C), indicating that they had the same mitochondrial membrane potential. Heat treatment $\left(42^{\circ} \mathrm{C}\right.$ for $\left.10 \mathrm{~min}\right)$ had no significant impact on mitochondrial membrane potential in both the WT 

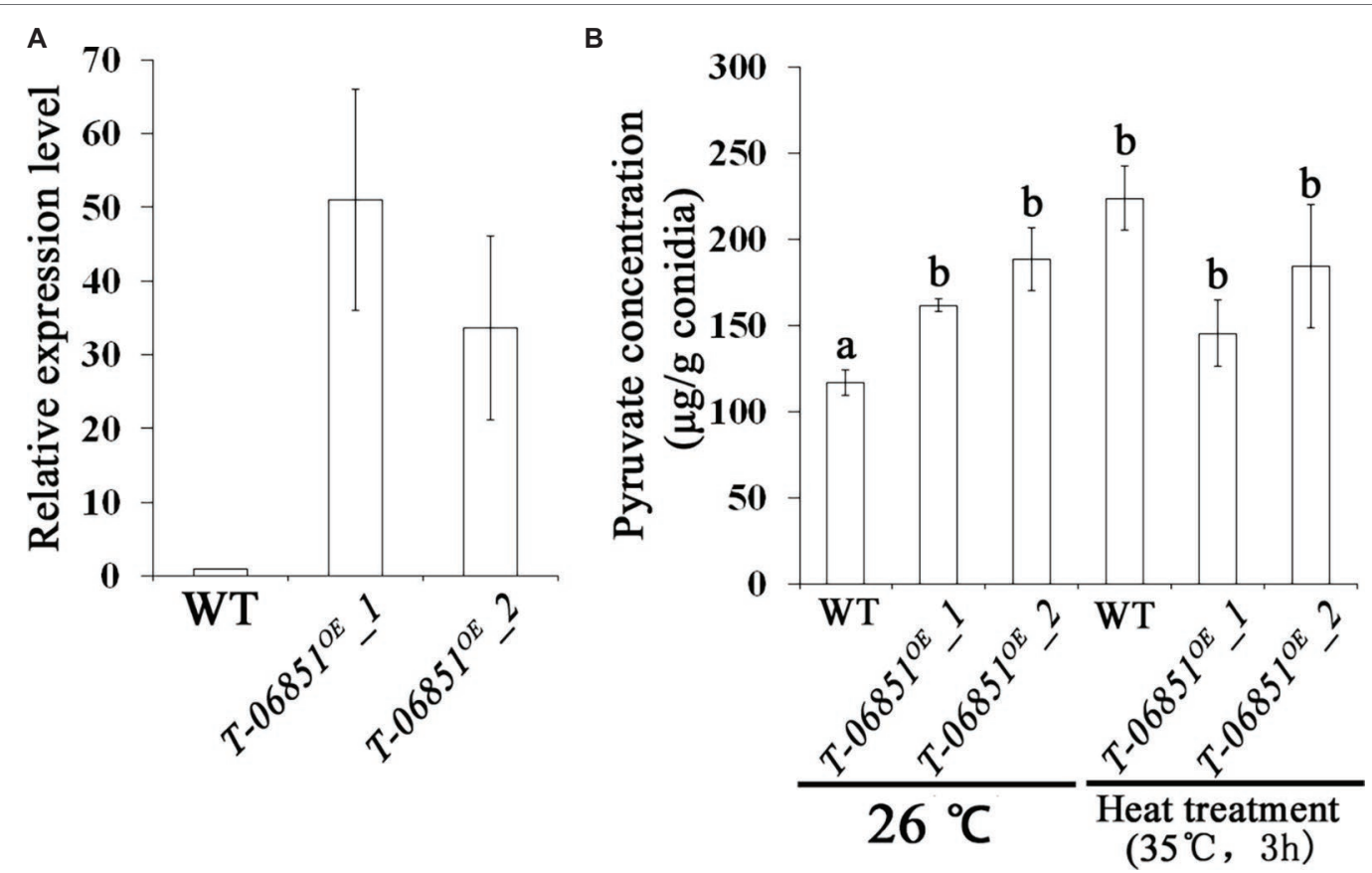

FIGURE 1 | Increasing pyruvate concentration in M. robertsii conidia by overexpressing the pyruvate kinase gene MAA_06851. (A) qRT-PCR confirmation of the overexpression of MAA_06851 in the conidia of two transformants (T-06851 ${ }^{O E_{-}} 1$ and $T-06851^{O E_{-}} 2$ ). The gene expression levels during conidiation in the two transformants ( 5 days after inoculation of conidia on PDA) were calculated relative to that of the wild-type (WT) strain, which was set to 1. (B) The pyruvate concentration in the conidia grown at optimal conditions $\left(26^{\circ} \mathrm{C}\right.$ on PDA for 14 days), and in the heat-treated conidia. Data are expressed as mean \pm standard error (SE). Values with different letters are significantly different ( $p<0.05$, Tukey's test in one-way ANOVA). All assays were repeated three times with three replicates per repeat.

strain and the transformants (Figure 2B). However, after heat treatment at $42^{\circ} \mathrm{C}$ for $30 \mathrm{~min}$, the ratio of green/red fluorescence intensity in the WT conidia was significantly greater than the transformants ( $p<0.05$, Tukey's test in one-way ANOVA) (Figures 2B,C); the ratio of green/red fluorescence intensity in the heat treated transformants was not different from those grown under the optimal conditions, indicating that the heat treatment induced a significant mitochondrial membrane potential collapse in WT but had no significant impact on the transformants.

\section{Increasing the Pyruvate Concentration Improved Conidial Tolerance to Heat Stress}

The impact of the elevated production of conidial pyruvate on abiotic stress tolerance was further assayed. Under optimal conditions [grown in the nutrient-rich medium 1/2 SDY (Sabouraud dextrose broth supplemented with $1 \%$ yeast extract) at $26^{\circ} \mathrm{C}$ ], the $\mathrm{GT}_{50}$ [time (hours) required for $50 \%$ of conidia to germinate] value of the WT strain was not significantly different from the two transformants (T-MAA_06851 ${ }^{O E}-1$ and T-MAA_06851 $1_{-2}^{O E}(p>0.05$, one-way ANOVA $)$, and the transformants were not significantly different from each other ( $p>0.05$, one-way ANOVA). The relative germination inhibition (defined in the Materials and Methods section) was used to compare the conidial germination of the WT strain and the transformants under several abiotic stresses. No significant differences in tolerance to UV radiation, osmotic stress, and oxidative stress were found between the WT strain and the two transformants ( $p>0.05$, one-way ANOVA; Table 1). However, the conidia of the two transformants treated with heat at $40^{\circ} \mathrm{C}$ for $60 \mathrm{~min}$ or at $42^{\circ} \mathrm{C}$ for $30 \mathrm{~min}$ germinated significantly faster than that of the heat-treated WT strain (Table 1), but at $36 \mathrm{~h}$, after the heat treated conidia were incubated at the optimal temperature $\left(26^{\circ} \mathrm{C}\right)$, nearly all conidia germinated.

The pathogenicity was assayed on Drosophila melanogaster adult flies. Inoculations were conducted by topically applying conidia onto the insect cuticle. No significant difference in virulence was found among the WT strain $\left[\mathrm{LT}_{50}\right.$ (time taken to kill $50 \%$ of insects $)=12.1 \pm 0.21$ days] and the two transformants $\left(T-M A A \_06851^{O E}-1: \mathrm{LT}_{50}=12.3 \pm 0.35\right.$ days; $T-M A A \_06851^{O E}-2$ : $\mathrm{LT}_{50}=12.6 \pm 0.41$ days; $p>0.05$, Tukey's test in one-way ANOVA).

\section{Discussion}

Heat treatment induces a rapid ROS burst in the conidia of $M$. robertsii, and the ROS level remains high until the ROS scavenger pyruvate is upregulated (Zhang et al., 2017). In the present study, we constructed two transformants that produce conidia under optimal conditions with elevated pyruvate concentration. This inhibited the rapid ROS burst induced by heat and prevented the collapse of the mitochondrial membrane potential, thereby increasing conidial tolerance to heat stress.

Fungal cells exposed to a mild stress develop tolerance not only to higher doses of the same stress but also to other stresses (Hohmann and Mager, 2003). This phenomenon is called cross protection. In the hyphae of $M$. robertsii, pyruvate 

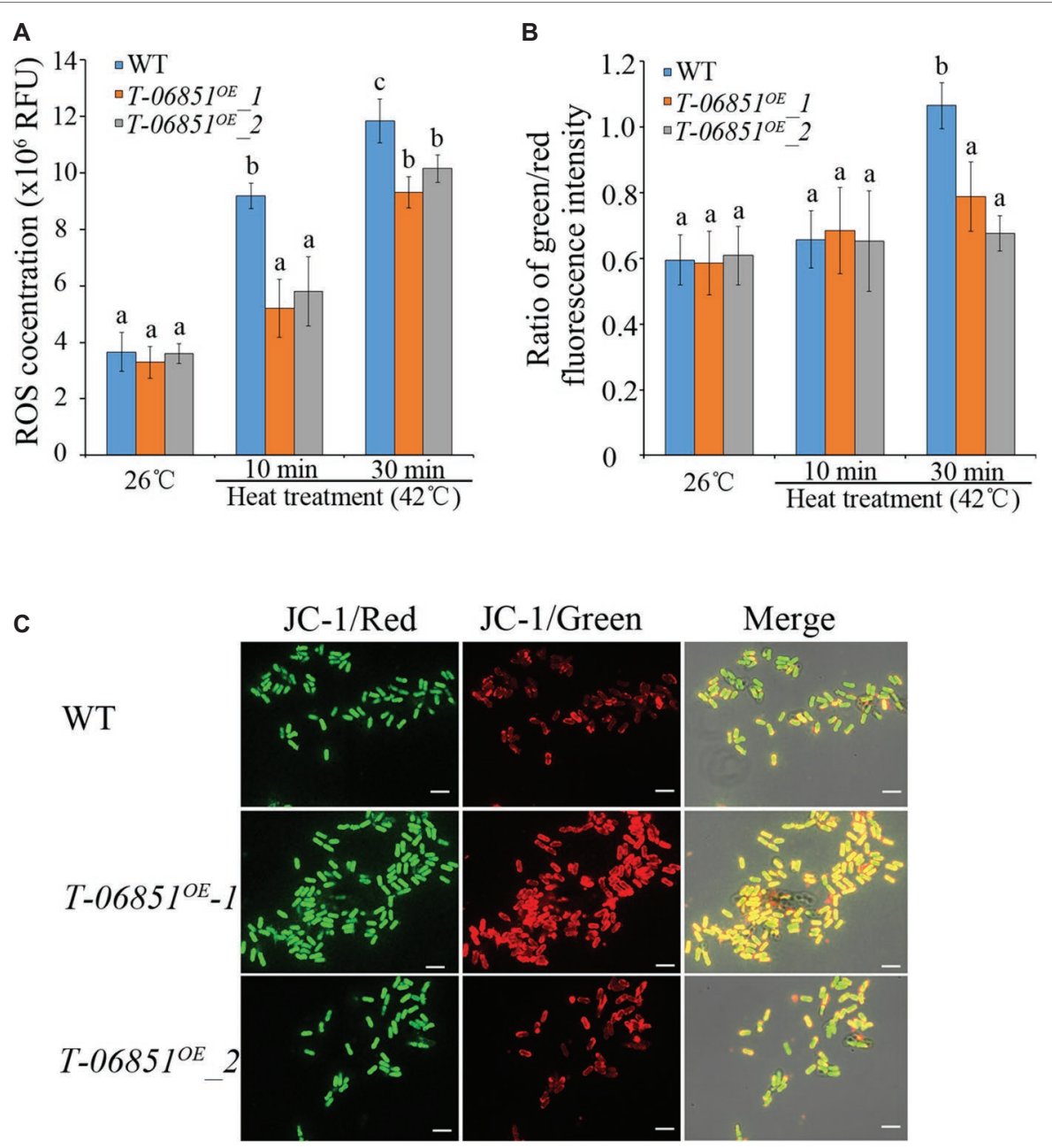

FIGURE 2 | Increasing pyruvate concentration reduces the ROS level and prevents the collapse of mitochondrial membrane in conidia treated with heat $\left(42^{\circ} \mathrm{C}\right.$, a widely used temperature for assaying heat stress tolerance of $\mathrm{M}$. robertsii conidia). (A) ROS levels in the conidia at the optimal temperature (26 ${ }^{\circ} \mathrm{C}$ ) and the heattreated conidia. RFU: relative fluorescence units. (B) Pyruvate prevents the collapse of mitochondrial membrane potential. The green/red fluorescence intensity ratio represents the extent to which the mitochondrial membrane potential collapses. (C) Typical pictures representative of five repeats of JC-1 staining for analysis of the mitochondrial membrane potential collapses. Bars, $10 \mu \mathrm{m}$. Values with different letters are significantly different (Tukey's test in one-way ANOVA, $p<0.05)$. WT: The wild-type strain; $T-06851^{O E_{-}} 1$ and $T-06851^{O E_{-}}$: two independent transformants with elevated pyruvate concentrations in the conidia.

TABLE 1 | Relative germination inhibition of the WT strain and two transformants with elevated pyruvate concentration in the conidia under the four abiotic stresses.

\begin{tabular}{|c|c|c|c|c|c|}
\hline & \multirow[t]{2}{*}{ UV radiation } & \multirow[t]{2}{*}{ Hyperosmotic stress } & \multirow[t]{2}{*}{ Oxidative stress } & \multicolumn{2}{|c|}{ Heat stress } \\
\hline & & & & $40^{\circ} \mathrm{C}(60 \mathrm{~min})$ & $42^{\circ} \mathrm{C}(30 \mathrm{~min})$ \\
\hline WT & $0.22 \pm 0.02^{\mathrm{a}}$ & $0.30 \pm 0.06^{a}$ & $0.04 \pm 0.01^{a}$ & $0.23 \pm 0.009^{a}$ & $0.60 \pm 0.04^{a}$ \\
\hline$T-06851^{O E_{-}} 1$ & $0.14 \pm 0.02^{\mathrm{a}}$ & $0.33 \pm 0.07^{\mathrm{a}}$ & $0.03 \pm 0.01^{\mathrm{a}}$ & $0.12 \pm 0.013^{b}$ & $0.45 \pm 0.03^{b}$ \\
\hline$T-06851^{O E}-2$ & $0.18 \pm 0.03^{a}$ & $0.31 \pm 0.04^{\mathrm{a}}$ & $0.05 \pm 0.01^{a}$ & $0.14 \pm 0.006^{b}$ & $0.46 \pm 0.02^{b}$ \\
\hline
\end{tabular}

Within the same abiotic stress treatment, the values followed by different letters were significantly different ( $p<0.05$, Tukey's test in a one-way ANOVA). All assays were repeated three times with three replicates per repeat.

The numerical values in the table: the relative germination inhibition of a given stressor on each strain was calculated as (Gc-Gt)/Gc, where Gc and Gt denote the GT 50 [time (hours) taken for $50 \%$ of conidia to germinate] of the stressed and unstressed conidia, respectively.

accumulation contributes to the cross-protection against multiple abiotic stresses, including heat, oxidative, osmotic/salt stresses, and UV radiation, which are largely attributed to these four stressors inducing the accumulation of pyruvate that scavenges stress-induced ROSs (Zhang et al., 2018). However, in the present study, we found that increasing the pyruvate concentration did not improve conidial tolerance to oxidative and osmotic/ salt stresses and UV radiation. This discrepancy could have 
resulted from the difference in metabolic states or mechanisms for abiotic stress tolerance between the hyphae and conidia.

Although the expression of the pyruvate kinase gene was increased over 30-fold in the two analyzed transformants, the pyruvate concentration only increased by approximately 1.5 -fold. This is similar to the stress-induced pyruvate accumulation in hyphae. Pyruvate has metabolic and redox properties and is located at a key intersection in the network of metabolic pathways (Roudier and Perrin, 2009). The fold of increase (1.5-fold) in pyruvate caused by stresses and by the genetic modification of pyruvate metabolism could be the greatest extent that fungal cells can resist. Changes in pyruvate concentration, to a greater extent, could otherwise be lethal or impair the fungal growth (Biagini et al., 2001; Zhang et al., 2017).

In conclusion, increasing the conidial pyruvate concentration by overexpressing a pyruvate kinase gene improved the tolerance of the conidia of $M$. robertsii to heat stress.

\section{Materials and Methods Construction of Transgenic Strains With Increased Pyruvate Concentration in Conidia}

In order to construct antisense RNA plasmids to knock down two genes (MAA_02871and MAA_08787) involved in pyruvate consumption during the conidiation of $M$. robertsii, the promoter region (645 bp) of the Pks1 gene (MAA_07745) (Zeng et al., 2018) was used. Approximately $200 \mathrm{bp}$ of the DNA fragment corresponding to part of the coding sequence of a target gene was amplified by PCR using the high-fidelity Taq DNA polymerase (Toyobo, Japan) and inserted downstream of the promoter of the Pks1 gene in the plasmid pPK2-bar-GFP-Pro (Zeng et al., 2018) to produce the antisense RNA plasmids, which were subsequently transformed into WT Metarhizium robertsii mediated by Agrobacterium tumefaciens AGL-1 as described (Xu et al., 2014). M. robertsii ARSEF2575 was obtained from the Agricultural Research Service Collection of Entomopathogenic Fungi. The fungal transformants were selected based on their resistance to the herbicide glufosinate ammonium (Sigma-Aldrich, USA), which was achieved by the bar gene in the plasmids (Xu et al., 2014). The fungal transformants with herbicide resistance were grown on PDA plates at $26^{\circ} \mathrm{C}$ for 5 days, and the conidiating mycelium was collected for RNA extraction and quantitative RT-PCR analyses (described below) to screen for transformants with expression of target genes reduced. All PCR products were confirmed by sequencing. The primers used in the present study are summarized in Table 2.

In order to construct the plasmid overexpressing the pyruvate kinase gene (MAA_06851), its coding sequence was cloned by PCR using the high-fidelity Taq DNA polymerase (Toyobo, Japan) and inserted downstream of the $P k s 1$ 's promoter in the plasmid pPK2-bar-GFP-Pro (Zeng et al., 2018). The resulting plasmid pPK2-bar-GFP-P7745-06851 was subsequently transformed into the WT $M$. robertsii mediated by $A$. tumefaciens. The expression of MAA_06851 during conidiation on PDA plates was assayed as described for MAA_02871 and MAA_08787.

\section{RNA Preparation and Quantitative RT-PCR Analysis}

TRIzol reagent (Life Technologies, USA) was used to extract total RNA from conidia collected from the conidiating mycelium that was grown at $26^{\circ} \mathrm{C}$ on PDA plates for 5 days. Two genes (act and tef) were used as internal standards in the quantitative RT-PCR analyses (Fang and Bidochka, 2006). The relative normalized transcript level of a gene was computed using the $2^{-\Delta \Delta \mathrm{Ct}}$ method (Livak and Schmittgen, 2001). cDNA was synthesized using a ReverTra Ace qPCR RT Master Mix with a gDNA remover (Toyobo, Japan). The quantitative PCR was conducted using a Thunderbird SYBR qPCR Mix (no ROX) (Toyobo, Japan). These experiments were repeated three times with three replicates per repeat.

TABLE 2 | Primers used in this study.

\begin{tabular}{|c|c|c|}
\hline Primers & Sequences & Note \\
\hline P7745-5 & GGCCCGGGAGGGCACTCAAATCATA & Cloning the promoter of MAA_07745 \\
\hline P7745-3 & GGGATATCGTTGATCCGAAGGTTGC & \\
\hline 6851-ORF5 & GGTITAAAATGGCTGCTGCTCAACA & Cloning the genomic region of ORF of \\
\hline 6851-ORF3 & GGTTAAACTAAGCCTGGCCAATTC & \\
\hline 2781-RNAi5 & GGGATATCTTCTGCAGCCAGTCGGT & Cloning part of MAA_02781's ORF \\
\hline 2781-RNAi3 & GGGATATCCATGAAGATGCAAATGA & \\
\hline 8787-RNAi5 & GGGATATCTCTTCTCAAGGCTGCTA & Cloning part of MAA_08787's ORF \\
\hline 8787-RNAi3 & GGGATATCATGGTCAGGGCAAGAAT & \\
\hline 6851-qRT5 & GGCAATGCTATCACTGACGG & qRT-PCR analysis of MAA_06851 \\
\hline 6851-qRT3 & CAATGATACCACCAGCAGCC & \\
\hline 2781-qRT5 & СССТСAАACCACССАТGTTG & qRT-PCR analysis of MAA_02781 \\
\hline 2781-qRT3 & GTGATGCCCCATGTGATGTC & \\
\hline 8787-qRT5 & АCСАСTCСTGTCTTCTTCGG & qRT-PCR analysis of MAA_08787 \\
\hline 8787-qRT3 & GTAGGCACCATTGTACTGCG & \\
\hline Act5 & TCCTGACGGTCAGGTCATC & Reference gene for qRT-PCR analysis \\
\hline Act3 & CACCAGACATGACGATGTTG & \\
\hline Tef5 & AGGCTGACTGCGCTATTCTC & Reference gene for qRT-PCR analysis \\
\hline Tef3 & ACTTGGTGGTGTCCATCTTG & \\
\hline
\end{tabular}




\section{Quantification of Pyruvate and Total Ross in Conidia}

Determination of pyruvate and ROS concentrations in conidia was conducted as previously described (Zhang et al., 2017). Briefly, approximately $10^{8}$ conidial cells were treated with liquid nitrogen for $5 \mathrm{~min}$ to kill the conidia. The inactivated conidia were incubated at $26^{\circ} \mathrm{C}$ for $2 \mathrm{~h}$ in $0.01 \%$ Zygolase (Seikagaku Biobusiness, Japan) and 10\% snailase (Solarbio, China) to partially remove the cell walls. Then, the treated conidia were washed twice with phosphate-buffered saline (PBS) buffer, suspended in $400 \mu \mathrm{l}$ of PBS, and homogenized $(10 \mathrm{~min}$ at $65 \mathrm{~Hz})$ with ceramic beads (diameter $=1 \mathrm{~mm}$ ) in a grinder (Jinxing, Shanghai China). Afterward, the supernatant was subjected to pyruvate quantification using the Pyruvate Assay Kit (Abcam, USA). For quantification of total ROSs, the supernatant was diluted 500-fold with the PBS buffer, and $50 \mu \mathrm{l}$ of the diluted supernatant was subjected to ROS quantification using the Oxiselect in vitro ROS/RNS Assay Kit (Cell Biolabs, Inc., USA).

To prepare heat-treated conidia, 14 days old fungal cultures on PDA grown at $26^{\circ} \mathrm{C}$ were incubated at higher temperatures for different amounts of time, and conidia were then collected for pyruvate and ROS assays. As previously described (Zhang et al., 2017), the conidia for pyruvate assays were treated at $35^{\circ} \mathrm{C}$. For ROS assays, $42^{\circ} \mathrm{C}$ was used for heat treatment.

All quantification assays were repeated three times with two replicates per repeat.

\section{Assays of Mitochondrial Membrane Potential}

The mitochondrial membrane potential of conidia was assayed with the JC-1 Staining Kit (Beyotime Biotechnology, Shanghai, China). Briefly, conidia were stained in the JG-1 (a mitochondrial membrane potential-dependent dye) staining solution for $30 \mathrm{~min}$, and then the conidia were rinsed three times with the staining buffer. The fluorescence intensities at wavelengths of 490 (excitation) and 530 (emission) $\mathrm{nm}$ and at 525 (excitation) and 590 (emission) nm were measured by a confocal microscopy (Zeiss, Germany), and their ratio was calculated with the ImageJ software (NCBI). The experiments were repeated five times with three replicates per repeat.

\section{Assays of Tolerance to Abiotic Stresses}

Assays of conidial tolerance to UV radiation, oxidative stress, hyperosmotic stress, and heat stress were conducted as previously described (Zeng et al., 2017, 2018). Conidia were collected from the fungal cultures that were grown at $26^{\circ} \mathrm{C}$ on PDA plates for 14 days. For the assay of tolerance to UV radiation,

\section{REFERENCES}

Biagini, G. A., Park, J. H., Lloyd, D., and Edwards, M. R. (2001). The antioxidant potential of pyruvate in the amitochondriate diplomonads Giardia intestinalis and Hexamita inflata. Microbiology 147, 3359-3365. doi: 10.1099/00221287-147-12-3359

Fang, W., Azimzadeh, P., and St. Leger, R. J. (2012). Strain improvement of fungal insecticides for controlling insect pests and vector-borne diseases. Curr. Opin. Microbiol. 15, 232-238. doi: 10.1016/j.mib.2011.12.012 the conidia were exposed to a 312-nm (280-320 nm) UV-B wavelength at $0.2 \mathrm{~J} \mathrm{~cm}^{-2}$ in a Bio-Sun++ chamber (VilberLourmat, Marne-la-Vallée, France). Then, the irradiated conidia were incubated at $26^{\circ} \mathrm{C}$, and the conidial germination was observed every $2 \mathrm{~h}$ using an inverted microscope (Leica, Germany).

For hyperosmotic and oxidative stress, tolerance was assayed by measuring the germination rate of the conidia in $1 / 2$ SDY supplemented with $0.75 \mathrm{M}$ of $\mathrm{KCl}$ or $3 \mathrm{mM}$ of $\mathrm{H}_{2} \mathrm{O}_{2}$, respectively.

The tolerance of conidia to heat stress was investigated by incubating the conidial suspensions at 40 or $42^{\circ} \mathrm{C}$ for a certain period of time $(10,30$, and $60 \mathrm{~min})$, and transferring to $26^{\circ} \mathrm{C}$ to continue growth. The germination was checked every $2 \mathrm{~h}$. The optimal temperature $\left(26^{\circ} \mathrm{C}\right)$ was used as a control.

The relative germination inhibition of a given stressor on each strain was calculated as (Gc-Gt)/Gc (Wang et al., 2014), where $\mathrm{Gc}$ and $\mathrm{Gt}$ denote the $\mathrm{GT}_{50}$ [time (hours) required for $50 \%$ conidia to germinate] of the stressed and unstressed conidia, respectively. All assays were repeated three times with three replicates per repeat.

\section{Pathogenicity Assays}

Pathogenicity assays were conducted as described (Wang et al., 2017). Inoculations were performed by immersing flies in conidial suspensions followed by vortexing gently for $10 \mathrm{~s}$. D. melanogaster ( $w^{1118}$ stock number 5905) was maintained at $27^{\circ} \mathrm{C}, 85 \%$ humidity, on food made without Tegosept and propionic acid. The recipe for fly food can be found at http:// cshprotocols.cshlp.org/content/2014/9/pdb.rec081414.full?text_ only=true. We bioassayed three tubes of 30 adult flies (aged 2-4 days) with a conidial suspension $\left(2.5 \times 10^{4}\right.$ conidia $/ \mathrm{ml}$ of water) produced from 14 days old plates. Control flies were treated with water alone. Mortality was recorded every $12 \mathrm{~h}$. All bioassays were repeated three times. $\mathrm{LT}_{50}$ values were calculated using the SPSS program (Chicago, USA).

\section{AUTHOR CONTRIBUTIONS}

$\mathrm{CW}$ and $\mathrm{XZ}$ performed the experiment. $\mathrm{XZ}$ and $\mathrm{WF}$ conceived the idea of the study. WF wrote the manuscript.

\section{FUNDING}

This work was supported by National Key R\&D Program of China (2017YFD0200400), and the National Natural Science Foundation of China (31672078). 
Hohmann, S., and Mager, W. H. (2003). Yeast stress responses. Berlin: SpringerVerlag.

Li, Z. Z., Alves, S. B., Roberts, D. W., Fan, M. Z., Delalibera, I., Tang, J., et al. (2010). Biological control of insects in Brazil and China: history, current programs and reasons for their successes using entomopathogenic fungi. Biocontrol Sci. Tech. 20, 117-136. doi: 10.1080/09583150903431665

Liao, X., Lu, H. L., Fang, W., and St. Leger, R. J. (2014). Overexpression of a Metarhizium robertsii HSP25 gene increases thermotolerance and survival in soil. Appl. Microbiol. Biotechnol. 98, 777-783. doi: 10.1007/s00253-013-5360-5

Livak, K. J., and Schmittgen, T. D. (2001). Analysis of relative gene expression data using real-time quantitative PCR and the $2^{-\Delta \Delta \mathrm{Ct}}$ method. Methods 25, 402-408. doi: 10.1006/meth.2001.1262

Rangel, D. E., Braga, G. U., Fernandes, E. K., Keyser, C. A., Hallsworth, J. E., and Roberts, D. W. (2015). Stress tolerance and virulence of insect-pathogenic fungi are determined by environmental conditions during conidial formation. Curr. Genet. 61, 383-404. doi: 10.1007/s00294-015-0477-y

Roudier, E., and Perrin, A. (2009). Considering the role of pyruvate in tumor cells during hypoxia. Biochim. Biophys. Acta 1796, 55-62. doi: 10.1016/j.bbcan.2009.02.003

Wang, J. B., Lu, H. L., and St. Leger, R. J. (2017). The genetic basis for variation in resistance to infection in the Drosophila melanogaster genetic reference panel. PLoS Pathog. 13:e1006260. doi: 10.1371/journal.ppat.1006260

Wang, X., Perez, E., Liu, R., Yan, L. J., Mallet, R. T., and Yang, S. H. (2007). Pyruvate protects mitochondria from oxidative stress in human neuroblastoma SK-N-SH cells. Brain Res. 1132, 1-9. doi: 10.1016/j.brainres.2006.11.032

Wang, J. J., Qiu, L., Cai, Q., Ying, S. H., and Feng, M. G. (2014). Three $\alpha-1,2$-mannosyltransferases contribute differentially to conidiation, cell wall integrity, multistress tolerance and virulence of Beauveria bassiana. Fungal Genet. Biol. 70, 1-10. doi: 10.1016/j.fgb.2014.06.010

Xie, X. Q., Wang, J., Huang, B. F., Ying, S. H., and Feng, M. G. (2010). A new manganese superoxide dismutase identified from Beauveria bassiana enhances virulence and stress tolerance when overexpressed in the fungal pathogen. Appl. Microbiol. Biotech. 86, 1543-1553. doi: 10.1007/ s00253-010-2437-2

Xu, C., Zhang, X., Qian, Y., Chen, X., Liu, R., Zeng, G., et al. (2014). A high-throughput gene disruption methodology for the entomopathogenic fungus Metarhizium robertsii. PLoS One 9:e107657. doi: 10.1371/journal. pone. 0115300

Ying, S. H., and Feng, M. G. (2011). Integration of Escherichia coli thioredoxin (trxA) into Beauveria bassiana enhances the fungal tolerance to the stresses of oxidation, heat and UV-B irradiation. Biol. Control 59, 255-260. doi: 10.1016/j.biocontrol.2011.07.005

Zeng, G., Chen, X., Zhang, X., Zhang, Q., Xu, C., Mi, W., et al. (2017). Genome-wide identification of pathogenicity, conidiation and colony sectorization genes in Metarhizium robertsii. Environ. Microbiol. 19, 3896-3908. doi: 10.1111/1462-2920.13777

Zeng, G., Zhang, P., Zhang, Q., Zhao, H., Li, Z., Zhang, X., et al. (2018). Duplication of a Pks gene cluster and subsequent functional diversification facilitate environmental adaptation in Metarhizium species. PLoS Genet. 14:e1007472. doi: 10.1371/journal.pgen.1007472

Zhang, X., St. Leger, R. J., and Fang, W. (2017). Pyruvate accumulation is the first line of cell defense against heat stress in a fungus. MBio 8:e01284-17. doi: 10.1094/PDIS-04-17-0573-RE

Zhang, X., St. Leger, R. J., and Fang, W. (2018). Stress-induced pyruvate accumulation contributes to cross protection in a fungus. Environ. Microbiol. 20, 1158-1169. doi: 10.1111/1462-2920.14058

Zhao, H., Lovett, B. R., and Fang, W. (2016). Genetically engineering entomopathogenic fungi. Adv. Genet. 94, 137-163. doi: 10.1016/bs. adgen.2015.11.001

Conflict of Interest Statement: The authors declare that the research was conducted in the absence of any commercial or financial relationships that could be construed as a potential conflict of interest.

Copyright () $2019 \mathrm{Wu}$, Zhang and Fang. This is an open-access article distributed under the terms of the Creative Commons Attribution License (CC BY). The use, distribution or reproduction in other forums is permitted, provided the original author(s) and the copyright owner(s) are credited and that the original publication in this journal is cited, in accordance with accepted academic practice. No use, distribution or reproduction is permitted which does not comply with these terms. 